\title{
XMM-Newton Observations of Hot Gas in Low Mass Dwarf Galaxies
}

\author{
Fabian Walter ${ }^{1}$, Jürgen Kerp ${ }^{2}$, Meikel Kappes ${ }^{2}$ \\ ${ }^{1}$ NRAO, P.O. Box O, Socorro, NM 87801, USA \\ ${ }^{2}$ University of Bonn, Auf dem Hügel 71, 53121 Bonn, Germany
}

\begin{abstract}
We present first results of our XMM-Newton study of the nearby dwarf galaxies IC 2574, Holmberg I and Sextans A. Soft X-ray emission has been detected in IC 2574 and X-ray point sources (presumably $\mathrm{X}$-ray binaries) are detected in all three galaxies.
\end{abstract}

\section{Results}

IC 2574, Holmberg I and Sextans A are part of our multi-frequency campaign to study the physical conditions of the interstellar medium (ISM) of dwarf galaxies. This sample was chosen because of the presence of prominent, supergiant HI holes in their ISM which are considered to be tracers of past massive star formation. According to the 'standard model' of the evolution of the ISM, diffuse soft X-ray emission is expected to originate from inside the $\mathrm{HI}$ holes. Besides a few pathological cases showing strong starbursts (e.g., NGC 3077, Ott et al. 2003; NGC 1569, Martin et al. 2002; NGC 4449, Summers et al. 2003), previous $\mathrm{X}$-ray missions have failed to detect this coronal gas phase in more typical dwarf galaxies beyond the Magellanic Clouds. Here we present first XMM-Newton maps of our dwarf galaxy sample. We find evidence for faint diffuse soft X-ray emission in IC 2574 (temperature: $\sim 0.5 \mathrm{keV}$, equivalent to $\sim 6 \times 10^{6} \mathrm{~K}$ ). We also present a comparison of the X-ray emission with high-resolution VLA HI observations and UV data obtained by the XMM-Newton optical monitor (OM). In IC 2574, more than $10 \mathrm{X}$-ray sources were detected; the most luminous source is associated with the center of a prominent supergiant HI hole (Walter et al. 1998; Kerp et al., in prep.). Some X-ray point sources (mostly X-ray binaries) were also detected within the HI distribution of the other 2 galaxies.

\section{References}

Martin, C. L., Kobulnicky, H. A., \& Heckman, T. M. 2002, ApJ, 574, 663

Ott, J., et al. 2001, AJ, 122, 3070

Ott, J., Martin, C. L., \& Walter, F. 2003, ApJ, 594, 776

Skillman, E. D., et al. 1988, A\&A, 196, 31

Summers, L. K., et al. 2003, MNRAS, 342, 690

Walter, F., Kerp, J., Duric, N., Brinks, E., \& Klein, U. 1998, ApJ, 502, L143

Walter, F., \& Brinks, E. 1999, AJ, 118, 273 


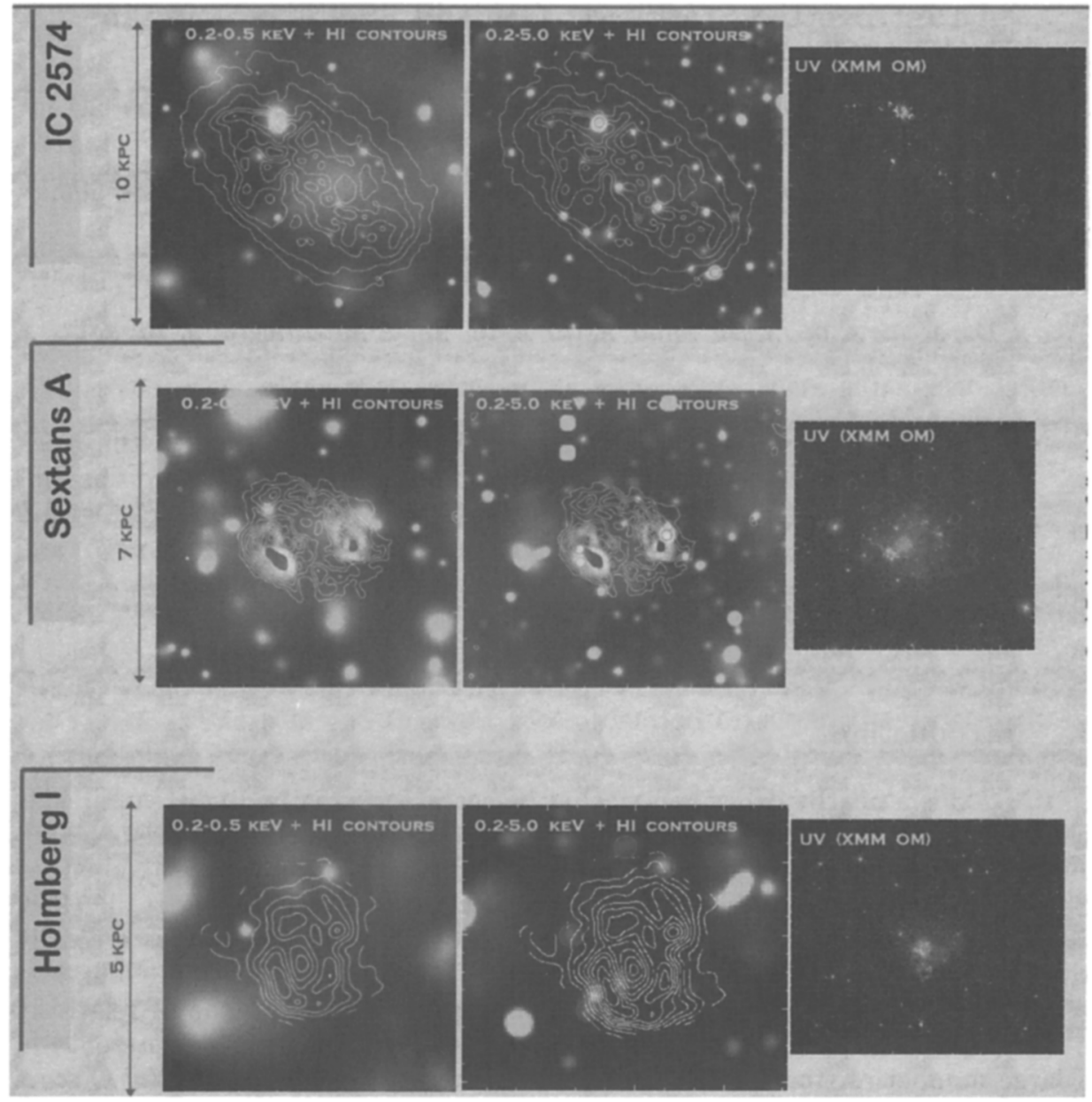

Figure 1. XMM-Newton images of Top: IC 2574; Middle: Sextans A; Bottom: Holmberg I. The left column shows the adaptively smoothed image of the soft X-ray emission $(0.2-0.5 \mathrm{keV})$. The brightest point sources have been removed to emphasize the diffuse emission. The contours represent the HI distribution in these systems (IC 2574: Walter \& Brinks 1999; Sextans A: Skillman et al. 1988; Holmberg I: Ott et al. 2001). Middle column: adaptively smoothed 'total' X-ray emission $(0.2-5 \mathrm{keV})$. The X-ray point sources within the HI extent of each galaxy are marked by circles (only point sources coincident with the HI distribution are considered here). Right column: Position of $\mathrm{X}$-ray point sources coincident with the HI distribution overlaid on an UV image of the galaxies (XMM OM). Few optical counterparts are identified. 